\title{
Strategie und Taktik optimaler Preisbildung im Dienstleistungssektor
}

\author{
Prof. Dr. rer. pol. Thomas Biermann
}

\section{Grundllagen der Preisgestaltung}

\subsection{Preisbildung im Wettbewerb}

Keine andere Einzelmaßnahme hat so durchschlagende und unmittelbare Wirkung auf den Ertrag wie eine Änderung der Preise. Die eigene Preispolitik und die der Wettbewerber steht zu Recht im Mittelpunkt unternehmensinterner Aufmerksamkeit, insbesondere bei Absatzproblemen im Markt. Der Europäische Binnenmarkt läßt einen harten internationalen Wettbewerb entstehen. Die bisher an nationale Schutzzonen gewohnten Dienstleistungssektoren (Versicherungen, freie Berufe, Ausbildung, Teile der Verkehrswirtschaft) müssen sich auf veränderte Bedingungen einstellen. Gleichzeitig zieht sich die öffentliche Hand aus klassischen Servicebereichen als Anbieter oder Regulationsbehörde zuruick. Für Dienstleister und Industriebetriebe, die ihre Produkte durch zusätzliche Serviceleistungen profilieren wollen, folgen daraus bisher unbekannte Risiken und Chancen.

Verschärfter Wettbewerb muß nicht gleichbedeutend sein mit Verfall der Preise und Erlöse. In etlichen Branchen gibt es Preisspielräume nach oben. Zwar betonen die Verkaufsmitarbeiter gegenüber ihrer Geschäftsfuihrung stets, daß man zu teuer anbiete, doch kann diese Aussage nicht auch auf einem Fehlurteil beruhen? Über den Preis, der relativ leicht zu ändern ist, läßt sich kundenseitig in Verkaufsverhandlungen sehr viel intensiver diskutieren als über designbedingte Qualitätsmerkmale oder kapazitätsbedingte Lieferprobleme. Mit modernen preispolitischen Instrumenten läßt sich somit eine betriebswirtschaftlich tragfähige Briicke schlagen zwischen verkaufsorientiert niedriger und ertragsorientiert höherer Preisfestsetzung.

\subsection{Kosten und Preise der Dienstleistung}

Der Dienstleistungsmarkt bietet für preisstrategische Überlegungen ein interessantes Beobachtungsfeld. Für Dienstleister spielt die von vornherein „richtige“ Preisfixierung eine tendenziell bedeutsamere Rolle als für den Produzenten physischer Güiter. Die Nicht-Lagerbarkeit der Leistung macht späteren Verkauf zu billigeren Preisen unmöglich, war die ursprüngliche Forderung zu hoch angesetzt. Gleichzeitig ist die Kostenstruktur im personalintensiven Servicesektor weitgehend von fixen Kosten bestimmt, die nicht von der abgesetzten Leistungsmenge abhängen. Daraus folgt die Versuchung zu hektischen Preissenkungen, wenn der Absatz stockt oder die Konkurrenten die Zähne zeigen.

Zur Vermeidung ruinöser Konkurrenz war deshalb frïher eine starke Preisregulierung durch die öffentliche Hand iiblich, sei es mittels Angebot der Leistung durch staatliche Monopole (z. B. Eisenbahn, Post, Telefon) oder durch gesetzliche oder quasi-gesetzliche Vorgaben gegenüber den im Sektor tätigen privaten Anbietern (Taxibetriebe). Der Abbau der Regulationsmechanismen in immer mehr Wirtschaftszweigen zeigt das kreative Potential der Dienstleister vor allem aus den Verkehrs- und Telekommunikationsbranchen bei der Entwicklung neuer preispolitischer Werkzeuge. Reicht die Preisforderung

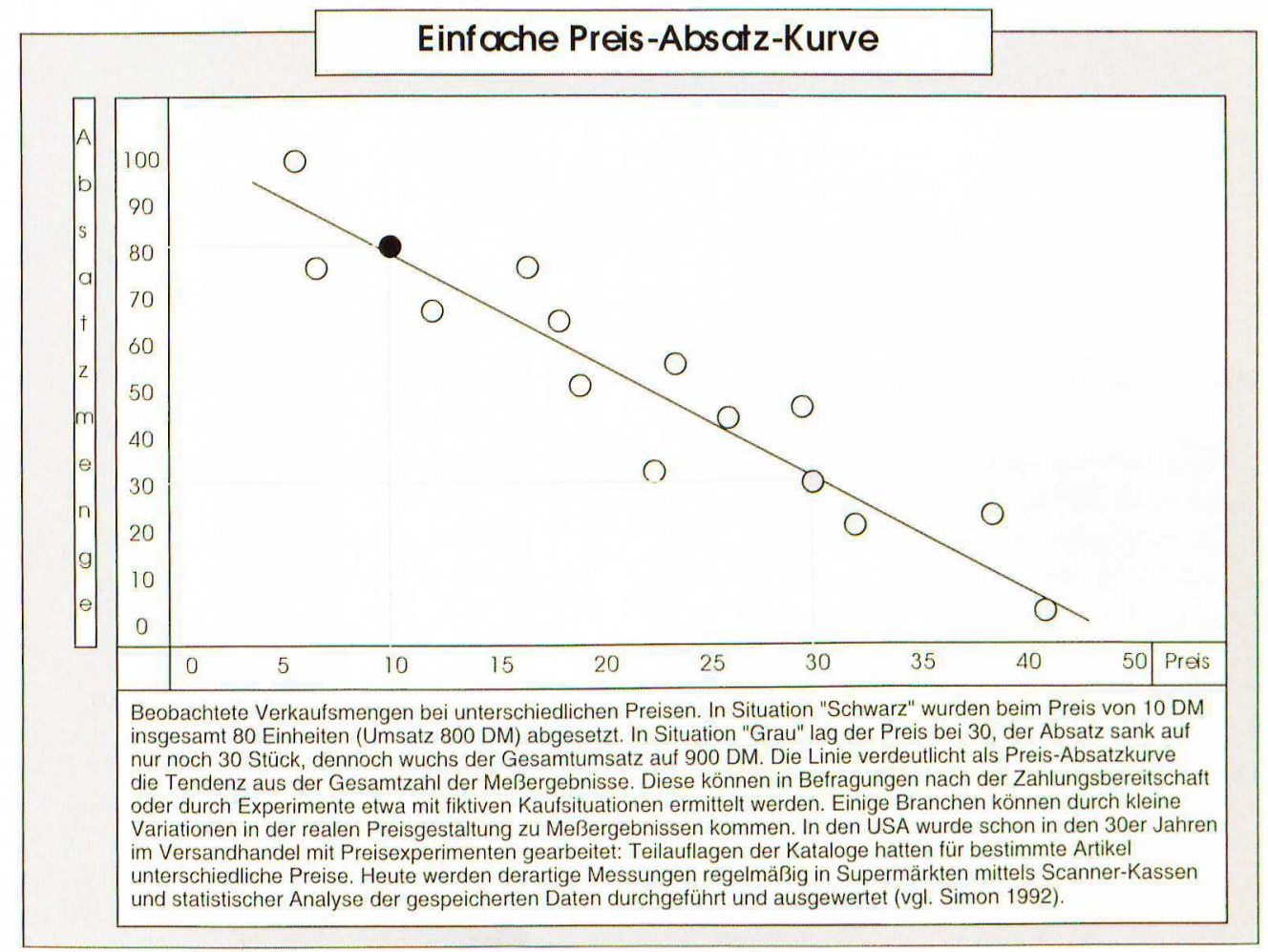


nicht zur Deckung aller zur Leistungserstellung notwendigen Kosten, entsteht dem Unternehmen ein Verlust; ist jedoch der Preis zu hoch angesetzt, verzichtet der Kunde auf den Kauf - also entsteht durch Einnahmeausfall und Ausschußproduktion ein noch höherer Verlust. „Richtig“ ist aus Unternehmenssicht der höchste Preis, bei dem der Kaufinteressent gerade noch zum Vertragsabschluß bewogen werden kann. Da ein Anbieter typischerweise mehreren hundert oder gar Millionen von potentiellen Kunden gegenübersteht, kann es einen einzigen in diesem Sinne richtigen Preis kaum geben. Unterschiedliche Käufer haben höchst unterschiedliche Zahlungsbereitschaften, in denen sich ihre subjektive Wertschätzung des Gutes, Einkommensverhältnisse oder Möglichkeiten des Ausweichens auf Alternativangebote widerspiegeln. Die Zahlungsbereitschaft ist dabei nicht einmal fuir einen einzelnen Nachfrager stabil, sie hängt von der jeweiligen persönlichen Situation zum Zeitpunkt der Kaufentscheidung ab. Der geforderten Geldzahlung wird der Nutzen gegenuibergestellt, den Produkt oder Dienstleistung dem Kunden versprechen.

\section{Das Prinzip der Preisdifferenzierung}

\subsection{Zahlungsbereitschaft der Nachfrage}

Naturgemäß sind nur wenige Unternehmen (etwa im spezialisierten Anlagenbau) in der Lage, die individuelle Preisobergrenze des einzelnen Kunden wirklich auszuloten - dies kann der Auktionator im Kunsthandel oder der Teppichhändler im orientalischen Basar besser. Die Grundregel für eine auf Ertragsverbesserung ausgerichtete Preisstrategie lautet daher; daß man sich durch differenzierte Preisfestlegung möglichst nahe an die unterschiedlichen „Reservationspreise“ der Nachfrager heranzutasten hat. Wenn A fuir mein Produkt maximal 12,DM hingeben will, B maximal 10,- DM und C nur 8,-DM, ist eine Mittelpreisstrategie mit Einheitspreis 10,-- DM offensichtlich suboptimal. Es werden zwei Einheiten abgesetzt, da C verzichtet, und ein Umsatz von DM 20,erzielt. Die Niedrigpreisvariante von einheitlich 8,- ergibt immerhin 24,- DM. Gelingt aber die vollständige Ausschöpfung des Kaufkraftpotentials, landen 30,- DM in der Kasse. Reine Preisdifferenzierung liegt vor, wenn eine vollkommen gleichartige Leistung von einem Hersteller zu unterschiedlichen Preisen abgegeben wird. Verbreitete Abstufungskriterien sind:

- Zeit,

- Ort,

- Menge,

- Personenmerkmale.

Zeitliche Differenzierung in Form von Saisonpreisen oder tageszeitlich gestaffelten Tarifen ist für viele Formen der Dienstleistung charakteristisch. Da kontinuierlich hohe Kapazitätsauslastung der entscheidende Ertragsfaktor ist, spielt nicht nur Neugewinn von Kunden uiber Billigtarife in nachfrageschwachen Zeiten eine Rolle, sondern ebenso die Verlagerung von Teilen der Nachfrage aus Spitzenlastzeiten in die saisonalen "Täler" (Peak Load Pricing).

Geschickt aufgebaute Preisdifferenzierung ist als solche kaum zu erkennen. Sie wird im Regelfall mit kleinen Unterschieden in der Produktgestaltung untermauert, die den Grundnutzen allenfalls unwesentlich verändern und die Kosten der Erstellung kaum berihren. Pionier dieser verschleierten Differenzierung waren Ende des vorigen Jahrhunderts die Eisenbahngesellschaften, die die Grundleistung Personentransport von A nach B in drei Klassen anboten. War die erste ausgesprochen luxuriös ausgestattet, stellte sich die dritte mit unbequemem Holzgestiihl dar, um kaufkräftigere Nachfrager zum Umsteigen auf das höherpreisige Produkt zu bewegen.

Die Feinabstimmung der Preise setzt detaillierte Marktkenntnis voraus. Grundsätzlich muß als Basis von Preisdifferenzierung eine Marktsegmentierung anhand dreier Gesichtspunkte erfolgen:

- klar voneinander abgrenzbare Segmente,

- in sich einheitliche Segmente und

- Übersichtlichkeit der Einteilung.

Die Verletzung der erstgenannten Bedingung führt zur Kannibalisierung der höherpreisigen Produktgruppen durch Abwanderung der Käufer in die niedrigeren Stufen und damit zu Ertragsverfall. Hier haben es Dienstleistungsanbieter leichter als Produzenten physischer Giiter, deren Erzeugnisse in einem Billigmarkt erworben und an die Hochpreis-Klientel weitergereicht werden können. Automobilhersteller mit ihrer ausgefeilten länderspezifischen Differenzierung der Preise kennen diese Problematik in Form der sogenannten Re-Importe (Tacke 1989).

\subsection{Segmentspezifische Preiselastizitäten}

Als entscheidende Kenngröße wird die Preiselastizität der Kundengruppen gesehen, die sich aus der Preis-Absatz-Kurve ableitet. Sie gibt an, wie sich die Absatzmenge bei einer Preiserhöhung von 1\% verändert. Liegt die Elastizität bei -1, so wird der (negative) Mengeneffekt bei einer Preiserhöhung den (positiven) Preiseffekt gerade neutralisieren, der in DM gemessene Gesamtumsatz bleibt gleich. Da dieser Umsatz aber mit geringerer Verkaufsmenge realisiert wird, steigt der Gewinn durch Kosteneinsparungen an. Je höher die variablen im Vergleich zu den fixen Kosten sind, um so stärker ist in diesem Beispiel der Gewinneffekt.

Die Preiselastizität gibt deutliche Hinweise auf die Sinnhaftigkeit von Preismaßnahmen. Je dringender der Konsument auf ein Gut angewiesen ist und je weniger er auf Konkurrenzprodukte ausweichen oder seinen Bedarf zurüickstellen kann, um so starrer wird sein Reaktionsverhalten. Als unelastisch gilt zum Beispiel die Nachfrage nach Pharmazeutika mit $-0,44$. Ausgesprochen preissensibel dagegen sind Konsumgüter des täglichen Bedarfs wie Kaffee $-2,93$ bis $-7,21$; Softdrinks $-1,59$ bis $-4,43$ oder Papiertiicher $-3,08$ bis $-4,00$ jeweils gemessen am aktuellen Marktpreis (Angaben nach Simon 1992).

\subsection{Mehr-Komponenten Preise}

Ein in Dienstleistungsbranchen gern beschrittener Weg ist die Aufspaltung des Preises in eine Grundgebuihr (täglich, monatlich, jährlich) und einen Einzelpreis pro Einheit. Abonnements, pauschale Wartungsverträge, Club- 


\section{Beispiel Peak Load Pricing}

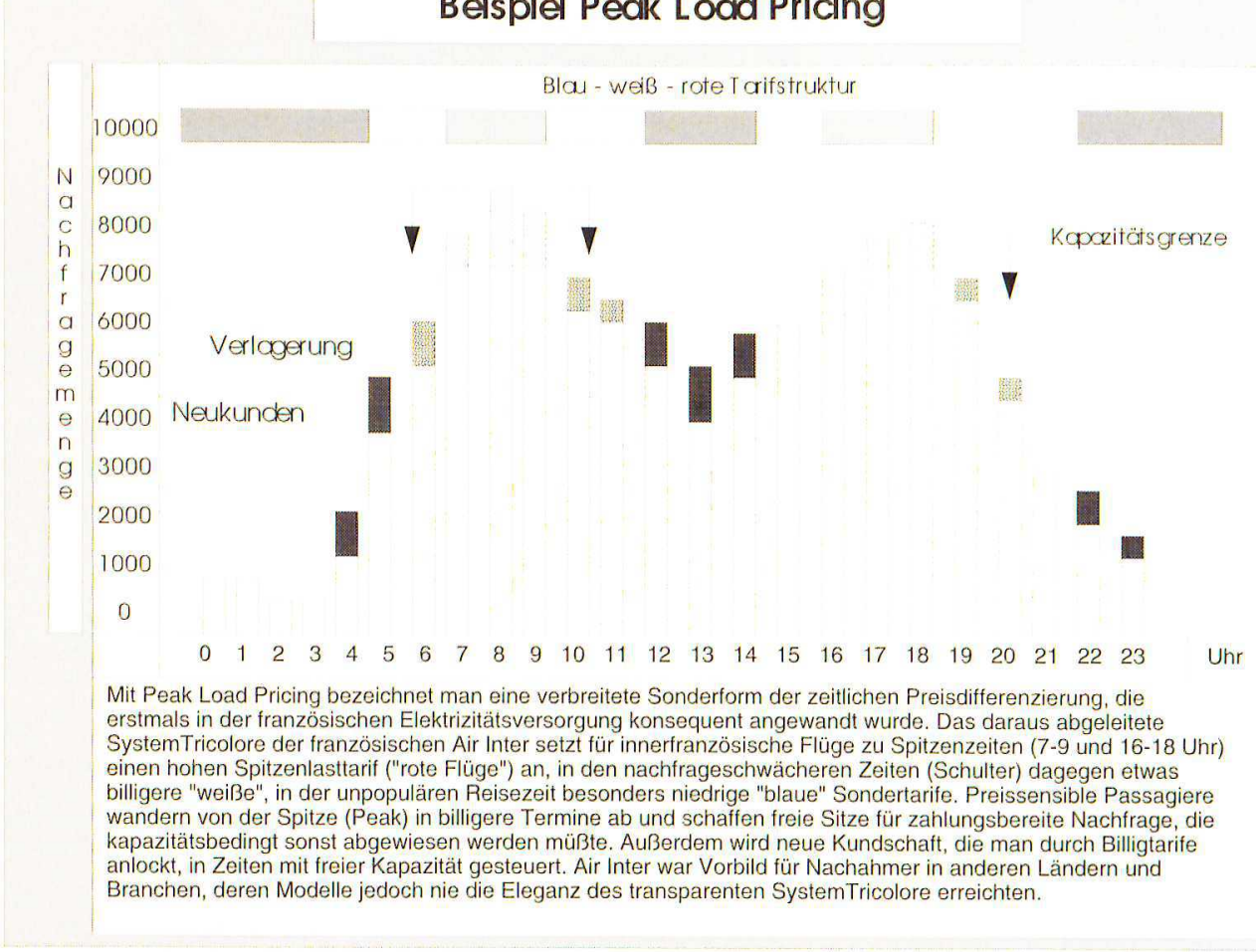

ausgesetzt ist. $\mathrm{Zu}$ unterscheiden ist zwischen der freigestellten Biindelung, bei der der Käufer die Elemente einzeln erwerben kann (Modulsystem), und der Zwangsbiundelung, die nur das Gesamtpaket zum Kauf stellt: in der Friihzeit der Computer etwa war eine IBMAnlage nur in Kombination mit einer Abnahmeverpflichtung fuir IBM-Lochkarten zu erhalten. Zwangsbuindelungen, welche die $\mathrm{Ab}$ hängigkeit der Kunden von einem Paketbestandteil ausnutzen, sind heute

mitgliedschaften (Autoclub, Buchclub), Kontoführungsgebiihren der Bank oder Kundenkartensysteme (BahnCard) sind verwandte Beispiele. Klassisches Modell ist der Telefontarif, der urspriinglich auf der Maxime „Dekkung der Infrastuktur-Fixkosten durch Grundgebiihren" basierte. Der Vorteil aus Anbietersicht besteht darin, daß der Kunde nach der grundsätzlichen Anschlußentscheidung die Fixkosten (seine Jahresgebiihr) nicht mehr in die Einzelkaufentscheidung einbezieht. Er nimmt so mehr Menge ab als bei einem uniformen Durchschnittstarif (z. B. keine Grundgebiihr, aber 1 DM pro Telefonat). Diese Art der Preisgestaltung fuihrt allerdings zum sogenannten Disneyland-Dilemma, erstmals am Beispiel eines Vergnïgungsparks (Oi 1971) wissenschaftlich untersucht: hohe Grundgebiihren (Eintritt in den Park) schrekken Nachfrager ab, die nur wenige Einzeleinheiten erwerben wollen (hier: wenige Attraktionen besichtigen); hohe Einzelpreise bei niedriger Grundgebiihr dagegen mindern die Absatzmenge bei den grundsätzlich kaufwilligen Gästen.

Auch hier steigert Preisdifferenzierung den Gewinn. Bei Mehr-Komponenten-Preisen kann die muihevolle Arbeit der Zuordnung zu Marktsegmenten dem Kunden sogar weitgehend selbst iiberlassen werden. Durch sein Verhalten und die darauf aufbauende Kaufentscheidung ordnet er sich selbst einem bestimmten Marktsegment zu, wenn verschiedene Kombinationen hohe Grundgebiihr/ niedriger Einzelpreis und umgekehrt zur Wahl gestellt werden. Dabei kann jeweils eine der Komponenten den Wert 0 annehmen.

\subsection{Bündelung}

Die gemeinsame Abgabe verschiedener Güter zu einem Gesamtpreis ermöglicht es, die eigene Stärke in einem Produktbereich zur Vermarktung von anderen Produkten zu nutzen, bei denen man uiberlegener Konkurrenz wettbewerbsrechtlich verpönt und selten geworden. Das Modulsystem dagegen bleibt populär. Gerade bei komplexen Problemlösungen, die Verfügbarkeit mehrerer Komponenten voraussetzen (Stereoanlage, Kleincomputersysteme, Golfausrüistung, Wintervorbereitung für das Auto), werden preisgiinstige Pakete als Einstiegshilfe vermarktet. Oft ist bei der werblichen Herausstellung beabsichtigt, daß der Kunde das Paketangebot als Diskussionsgrundlage für das Zusammenstellen eines anderen auf seine Beduirfnisse zugeschnittenen Sets mit höherwertigen Bausteinen macht (Entbündelung). Dabei darf es dann natiirlich preislich ruhig etwas mehr sein.

\subsection{Rückvergïtungen}

Wird dem Kunden zugesagt, daß er - bestimmtes Verhalten vorausgesetzt - einen Teil des Entgeltes später zurückerhält, kann dies ein Zusatzargument für unentschlossene Käufer sein und vor allem zur festen Kundenbindung beitragen. Frïher gab es Rabattmärkchen im Einzelhandel, heute sind Beitragsriickerstattungen bei Schadensfreiheit im Versicherungswesen üblich. Im Verhältnis Hersteller - Handel sind in vielen Branchen nachträgliche Mengenrabatte oder Umsatzboni zur Routine geworden. Anders als bei der Garantie, wo eine Ruickzahlung vom Verhalten des Produktes abhängt, hat bei der Rückvergütung der Kunde den Eindruck, daß er den Preis durch seine Aktionen (konkret: weitere Käufe) im Nachhinein selbst bestimmt.

Die Rüickverguitung kann statt in Geld auch in Wareneinheiten erfolgen, etwa mittels Gutscheinpunkten bei manchen Konsumguitern. Der wohl interessanteste Anwendungsfall sind die Frequent-Flyer-Programme der großen Fluggesellschaften, seit wenigen Jahren auch in Deutschland angeboten (,Miles and More“-Programm der Lufthansa). Wer eine bestimmte Zahl von Flugmeilen mit der entsprechenden Gesellschaft absolviert, erwirbt 
das Anrecht auf Freifliige. Die Idee stammt von American Airlines, die damit 1981 nach eigener Aussage das „wirksamste Marketinginstrument unserer Geschichte“ (nach Simon 1992) erfunden hat. Das Sammeln von Freimeilen wurde in den USA fast zum Nationalsport. Daß auf Firmenkosten reisende Geschäftsleute iiberfliissige Reisen oder meilenintensive Umwege eimplanen, um privat nutzbare Freifliige zu erwerben - ein immer wieder laut werdender Verdacht - ließ sich nie nachweisen, aber auch kaum mit völliger Sicherheit widerlegen. Trotz einiger Folgeprobleme fuir die Airlines (Handel mit Meilencoupons, ertragsstarke Fliige zur Urlaubssaison von Freigästen blockiert) erwies sich clas Modell als so erfolgreich, daß die anderen Fluggesellschaften ebenfalls derartige Kundenbindungssysteme installierten. Hotelketten und Autovermieter zogen nach. Schon die durch die Kunden mittels Coupon-Einreichung offengelegten Verhaltensdaten machen diese Systeme für den MarketingExperten wertvoll.

Zwar werden im Zweifelsfall die Kunden einen unmittelbaren Preisvorteil zum Kaufzeitpunkt einem Riickverguitungsversprechen vorziehen, doch lassen sich nach dem Frequent-Flyer-Vorbild mit etwas Kreativität auch in anderen Branchen interessante Systeme der Kundenbindung modellieren. Ruickverguitungen in Warenform haben aus Produzentensicht besonderen Charme, in einigen Fällen sind Kreuzcoupons eine überlegenswerte Alternative: bei Kauf einer Leistung (Beispiel Hotelübernachtung) erhält der Kunde einen Gutschein fuir die Leistung eines anderen Herstellers (zum Beispiel Mietwagen). Hier können sich nicht konkurrierende Anbieter mit gleichgearteter Kundschaft Bälle zuspielen, die Stammkundenbindung für den einen wird mit Neukundenansprache für den anderen verkniipft.

\section{Preisbildung in schwierigen Zeiten}

\subsection{Preiskämpfe}

Gerät ein Unternehmen in eine Absatzflaute, wird rasch der Ruf nach Preissenkungen laut. Vor allem das Verkaufspersonal wird in seiner Not immer wieder auf günstigere Angebote der Konkurrenz verweisen und Gegenmaßnahmen einfordern. Im Dienstleistungssektor ist der Druck auf das Preisniveau besonders ausgeprägt, aber ein Nachgeben gerade deshalb riskant. Der Weg des geringsten Widerstandes führt in den freien Fall: eine Preissenkung wird mit hoher Wahrscheinlichkeit von den Mitbewerbern mit noch weitergehenderen Absenkungen beantwortet. Sich nach unten drehende Preisspiralen sind zum Beispiel regelmäßig in Abhängigkeit von Konjunkturzyklen in Seeschiffahrt und Luftfracht zu beobachten. Für eine solche Entwicklung will kaum jemand die Verantwortung übernehmen. Um das Markenimage nicht zu beschädigen und keinen Preiskrieg loszutreten, wird nach hitziger Diskussion der Beschluß gefaßt, das Preisniveau unter dem Motto "da muissen wir durch" stabil zu halten. Gleichzeitig signalisiert die Führung bis zur Bewältigung der Absatzkrise die stillschweigende Duldung einer gewissen Flexibilität der Verkaufsmitarbeiter bei den Nicht-Preis Konditionen.

\subsection{Das Phänomen der Preistreppe}

Handelt es sich um eine vorïbergehende Branchenkrise, die alle Wettbewerber gleichermaßen trifft, ist diese Taktik vertretbar: Hat das Unternehmen aber in Wirklichkeit Schwächen beispielsweise in der Qualität oder dem relativen Preis-Leistungsverhältnis im Vergleich zu neuen Konkurrenten, droht ein Preistreppeneffekt: bei offiziell hoch gehaltenem Listenpreisniveau werden auf Nebenschauplätzen seitens des Verkaufs dem Kunden versteckte Verguinstigungen gewährt. Großziigige Interpretation von Mengenrabattregeln, iiber die Aktionsfrist reichende Sonderaktionspreise, Skonti, mehrfache Riickverguitungen, Freifrachten, Garantieausweitungen und die Verlängerung von Zahlungszielen lassen die tatsächlichen Zahlungseingänge stufenweise abrutschen. Womöglich werden selbst bekanntermaßen dubiose Zahler wieder gegen offene Rechnung beliefert. Unternehmensintern herrscht kaum Transparenz über die kumulativen Effekte dieses Erlösverfalls. Zwar merkt der Versandleiter; der Servicechef, der Finanzbuchhalter in seinem Bereich Kostensteigerungen, aber wer führt all diese Effekte zusammen? Der Controller kümmert sich in der beschriebenen Krisensituation erfahrungsgemäß nicht um Kleinbeträge im Konto „Boni und Skonti“, sondern konzentriert sich auf Schliisselfragen wie die Reduzierung der Stromverbrauchskosten in der Hauptverwaltung. Die effektiv erzielten Verkaufserlöse können unter Berïksichtigung der Dreingaben und Inkassoverzichte in der Praxis durchaus um 25-30\% unter die Rechnungsbeträge absinken (Marn/Rosiello 1993). Dieser Preistreppen- oder Netto-Netto-Effekt gewinnt seine besondere Brisanz dadurch, daß der buchhalterisch ausgewiesene Umsatz tatsächlich erst einmal steigt. Die Mehrbelastungen treten an vielen übersehenen Punkten auf, bei weitausholenden Garantieversprechen an Kunden oder Werbezuschußzusagen an den Handel oft erst mit einer Verzögerung von Monaten, vielleicht sogar ein bis zwei Jahren. Vor diesem Hintergrund gibt sich die Geschäftsführung auf Basis fakturierter Umsätze leicht der Illusion hin, die Politik stabiler Preise habe gegriffen und die Krise sei bewältigt. Die böse Überraschung folgt spätestens, wenn der Wirtschaftsprüifer eine Neubewertung der Rückstellungen für Garantien anmahnt oder die Zahlungsausfälle der leichtfertig bedienten faulen Kunden eine Durchleuchtung der Werthaltigkeit der Forderungsbestände nahelegen.

\section{Dynamische Preisgestaltung}

\subsection{Orientierung am Produktlebenszyklus}

Die Suche nach dem optimalen Absatzpreis ist so schwierig wie für einen Schützen das Anlegen auf ein bewegliches Ziel. Nicht nur das Nachfrageverhalten, auch das der Wettbewerber kann sich in Folge eigener preispolitischer Aktionen deutlich verändern. Erschwerend kommt hinzu, daß ein optimaler Preis nur fuir einen bestimmten Zeitraum gilt, in der Einfuihrungsphase eines Produktes oder einer Dienstleistung sind andere Maßstäbe anzulegen als in der Reifephase. Dynamisches Preismana- 
gement verlangt Kenntnis über die gegenwärtige Situation hinsichtlich Kosten der Erstellung, Zahlungsbereitschaft der Nachfrager, Differenzierungsmöglichkeiten und Konkurrenzverhalten, aber auch eine Prognose iiber zukuinftige Veränderungen. Zwei bewährte Hilfsmittel erleichtern diese Vorschau, die Erfahrungskurve und der Produktlebenszyklus.

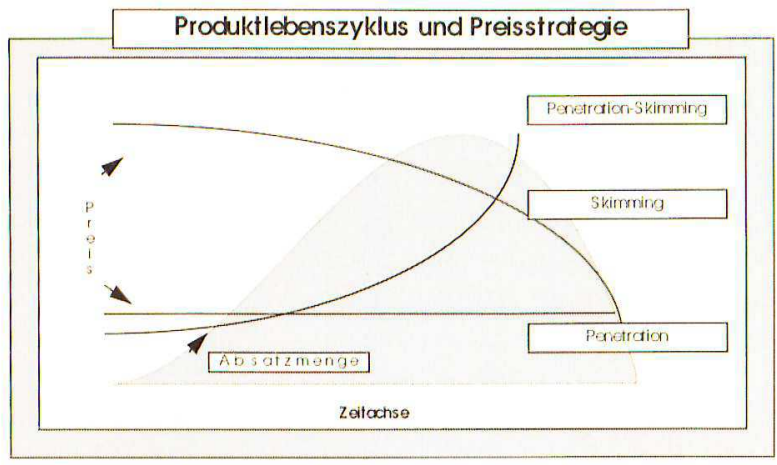

Die als Phänomen seit 1936 bekannte, aber erst ab 1966 so bezeichnete Erfahrungskurve geht auf Beobachtungen aus der Industrie zurück. Bei erhöhter Produktionsmenge sinken bekanntermaßen die Stïckkosten. Der Lernprozeß der Arbeiterschaft und die Optimierung von Produktionsprozessen fuihrt dazu, daß dieser Effekt auch bei kumulierten Mengen eintritt. Die Produktionskosten heute hängen also von den historischen Produktionsmengen der Vorperioden mit ab. Man rechnet mit einem Stiickkostenvorteil von $10-30 \%$ bei Verdoppelung der kumulierten Produktionsmenge. Empirische Untersuchungen (siehe Simon 1992) belegen diese sogenannte Lernrate fuir etliche Branchen, wobei der Effekt bei neuen Produkten stärker zum Tragen kommt (Integrierte Schaltkreise 27,8\%) als bei alteingefuihrten (Elektroherde $11,7 \%$ ). Es gilt daher als strategisch vorteilhaft, möglichst friih die Marktführerschaft bei innovativen Produkten und Leistungen zu erwerben, um einen später kaum noch einzuholenden Kostenvorteil vor der Konkurrenz zu erwerben.

Der Produktlebenszyklus zählt zu den wichtigsten Erklärungsmodellen des Marktverhaltens. Er unterstellt einen charakteristischen Verlauf der Akzeptanz und damit der Verkaufsmengen neuer Produkte. Die Einfuihrungsphase ist von schleppendem Absatz gekennzeichnet, nur sehr wenige innovativ eingestellte Kunden wagen sich an den ,neumodischen Kram" heran. Gerät das Produkt später in den Blickwinkel typischer Trendsetter - wohlhabende Konsumenten, fortschrittliche professionelle Nutzer - steigt die Absatzmenge mit zweistelligen Wachstumsraten an. Aus einer früher belächelten technischen Spielerei oder exotischen Serviceleistung wird ein Massenartikel, bis eine Sättigungsgrenze erreicht ist und die Verkaufsmengen schließlich abfallen.

\subsection{Ertrag und Marktanteil im Zielkonflikt}

Die strategischen Schlußfolgerungen aus beiden Phänomenen fuir die Preisgestaltung sind widersprïchlich. Soll man als Innovator mit niedrigen Preisen den Markt angreifen, um zu hohen Stückzahlen und damit zu den Kostenvorteilen aus dem Erfahrungskurveneffekt zu kommen? Oder muß man in der Einführungsphase hohe
Preise verlangen, um die Entwicklungskosten frihzeitig wieder hereinzuholen? Traditionell wurde mit Blick auf den Produktlebenszyklus die zweite Variante bevorzugt: die echten Pionierkunden erwerben Neuerungen ,koste, was es wolle", die Preiselastizität dieser FrühphasenKlientel ist gering. Dem preissensibleren Massenpublikum ist der Produktnutzen noch gar nicht zu vermitteln, sie steigen daher selbst bei günstigen Angeboten nicht ein. Die geschilderte Strategie ist als Abschöpfen (Skimming) bekannt. Die hohe Zahlungsbereitschaft der anspruchsvollen Avantgarde-Klientel wird mit hohen Preisen abgeschöpft, die durch das Vorbildverhalten der Avantgarde ermutigte Massennachfrage dann Zug um Zug mit Preisnachlässen angelockt. Der letzte Nachziigler wird schließlich im Super-Sonderangebots-Ausverkauf bedient - es findet klassische Preisdifferenzierung uiber die Zeitachse der Produktattraktivität statt.

Im Gegensatz dazu steht die Penetrations-Strategie, mit der japanische Hersteller von Motorrädern und Unterhaltungselektronik ab 1970 die Märkte in USA und Europa eroberten. Das neue Produkt wird trotz technischqualitativer Überlegenheit von vornherein preisgünstig angeboten, der Konsument zahlt keinen Mehrpreis für Pionierkäuferschaft. Eine erfolgreiche Penetrations-Strategie kann zur dramatischen Verküirzung der Produkteinfuihrungsphase führen, die lukrative Wachstumsphase ist schneller erreicht. Ihre Befürworter weisen außerdem daraufhin, daß so potentiellen Wettbewerbern der Markteintritt verleidet wird, während Skimming durch die hohen Anfangspreise preisaggressivere Nachahmer geradezu magisch anzieht. Außerdem besteht die Gefahr, daß ein Teil der anvisierten Kundschaft die Abschöpfungsabsicht erkennt und die Nachfrage in Erwartung sinkender Preise hinauszögert.

Eine generelle Aussage, welche strategische Grundausrichtung vorteilhafter ist, läßt sich kaum treffen. Wo hohe Preiselastizität der Nachfrage zu Beginn des Produktlebenszyklus eindeutig feststellbar ist, macht die aggressive Marktdurchdringung Sinn, wo der innovative Vorsprung gut abgesichert werden kann (etwa mittels Patenten) ist das Abschöpfen die bessere Alternative. Das juingste Modell dynamischer Preisgestaltung stellt eine Kombination beider Gedanken dar: bei PenetrationSkimming (Pen-Skim) wird eine aggressive Niedrigpreisstrategie vom Start weg zum Ausbau einer starken Marktposition genutzt, die später - wenn die Mitbewerber niedergerungen sind - Raum zu Preiserhöhungen läßt. Der ursprüngliche Ansatz der Abschöpfungsphilosophie, daß die Pioniere in der Kundschaft mit hohen Preisen die Entwicklungskosten der Innovation decken sollen, wird auf den Kopf gestellt.

\subsection{Carryover-Effekte}

Marktforscher aller Branchen versuchen, mit komplizierten ökonometrischen Verfahren den Bestimmungsgründen des Kaufverhaltens auf die Spur zu kommen. Preis, Qualität und Einkommen der potentiellen Kunden sind wichtige erklärende Variablen. Oft stellt sich jedoch für die Prognose der Absatzmenge $\mathrm{x}$ in der Periode $t$ die Variable $x_{t-1}$ als statistisch stärkste Einflußgröße heraus, das Kaufverhalten heute wird also von dem der Vor- 
periode weitgehend vorherbestimmt. Dieser sogenannte Carryover-Effekt findet seine Begründung einerseits im Wiederkauf, andererseits im Einfluß bisheriger Käufer auf Neunachfrage etwa durch Empfehlungen beziehungsweise gewollte oder ungewollte Produktdemonstration und Vorbildfunktion.

Bei starker persönlicher Bindung (Kosmetika), Geschmacksprodukten (Kaffee, Zigaretten) oder bei subjektiv empfundenem hohen Risiko der Nutzung unerprobter Produkte (Pharmazeutika) determinieren die Vorperioden-Käufe zu über $60 \%$ die aktuellen Umsätze. Leider ist der Carryover-Effekt im Servicesektor längst nicht so umfassend erforscht und quantifiziert wie bei Konsumgiitern, doch deuten alle verfuigbaren Daten darauf hin, daß er hier noch stärker ausgeprägt ist. Ein hoher Carryover-Koeffizient ist gleichbedeutend mit Markentreue und ein zusätzliches Argument für eine auf ziigige Marktdurchdringung angelegte Preisstrategie, gleichzeitig erlaubt er spätere Preisanhebungen, ohne daß uibermäßige Einbruiche in der Absatzmenge zu befürchten sind.

\subsection{Hardware plus Service}

Dynamische Preisgestaltung birgt besondere Chancen für Hersteller physischer Produkte, die ihre Palette an „Hardware" mit Dienstleistungsangeboten gegenüber dem Wettbewerb profilieren und zusätzliche Deckungsbeiträge erwirtschaften wollen. Die Entgeltgestaltung für die angekoppelte Serviceleistung kann bei geschickter Argumentation einen hohen Preis für das Grundprodukt iiberdecken. Mehrere Autohersteller werben mittlerweile fast ausschließlich mit dem günstigen Zinssatz ihrer Leasing-Finanzierung. Im Maschinen- und Anlagenbau ist vielfach eine modifizierte PenetrationSkimming Strategie anzutreffen: die Maschine wird preisguinstig offeriert, der Profit wird mit lukrativen
Wartungsverträgen und Ersatzteillieferungen gemacht. Eine solche Vorgehensweise kann den Wettbewerb auf die Einzelfelder verlagern, in denen das eigene Angebot qualitativ oder preislich besondere Vorteile bietet.

Leider kennt die Praxis viele Fälle, in denen sich ein Anbieter ohne systematisch durchdachte und später konsequent durchgehaltene Strategie im preispolitischen Instrumentarium seiner vielfältigen Grund- und Zusatzleistungen hoffnungslos verheddert hat. Die Einfuihrung von Zusatzleistungen fordert daher eine unzweideutige Standortbestimmung über die Durchsetzbarkeit von Preisforderungen fuir Zusatzservice beziehungsweise die konkrete Verbesserung in der Positionierung des Kernangebotes bei Zusatzservice zum Preise 0 (siehe Biermann 1994).

\section{Kapazitätsorientierte Preisgestaltung}

\subsection{Das Kapazitätsproblem im Dienstleistungs- unternehmen}

Industriefirmen, die ihre Produkte bei Nachfrageeinbriichen in Erwartung besserer Zeiten einlagern und den Bestand in der Bilanz aktivieren, duirfen die Preisdiskussion mit größerer Gelassenheit angehen als Dienstleister, deren Output nicht lagerfähig ist: das leere Hotelbett, der leere Passagiersitz im Flugzeug sind Ausschußproduktion ohne Verwertungsmöglichkeit. Dies macht den Servicesektor so anfällig gegenüber Preiskämpfen. Die Kehrseite der Medaille ist mindestens ebenso betriiblich: in Zeiten starken Kaufinteresses kann nicht auf Lagerbestände zurïckgegriffen werden, nur die tagesaktuelle Produktionsmenge steht zur Verfügung es kommt zur Nachfrageabweisung an die Konkurrenz. Vor dem Dilemma zwischen „Überpreis und Unterauslastung“ und „Unterpreis und Abweisung“ bewegt sich

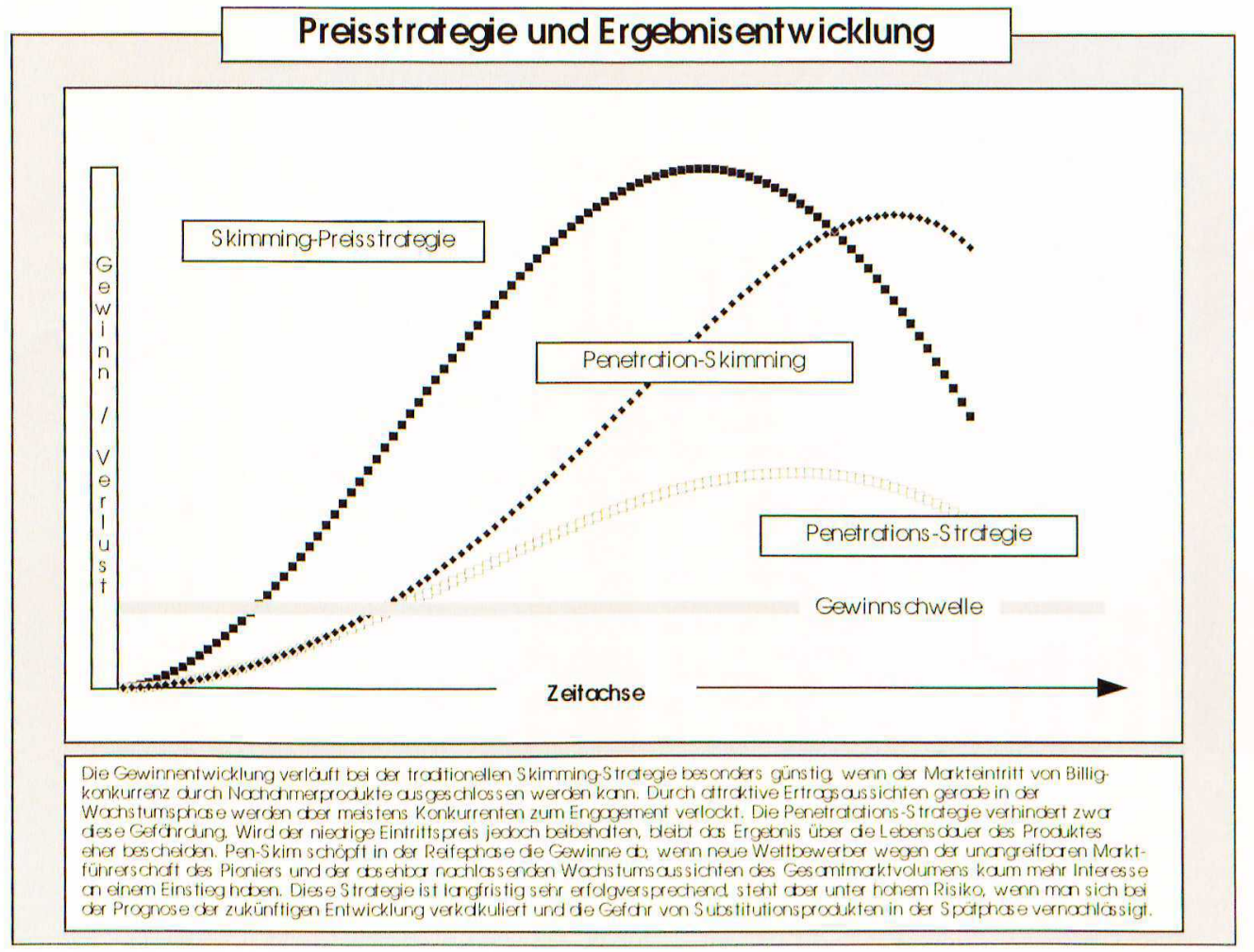

jeder Anbieter von Dienstleistungen. Preisdifferenzierung mittels Saisonangeboten oder Einzelaushandlung ist ein Schritt in die richtige Richtung, bei stark variierender Nachfrage ohne fest vorhersehbare Gesetzmäßigkeiten reicht er nicht aus. Hier hilft allein ein professionelles Yield Management, also eine exakte Steuerung der fuir verschiedene Entgelte verfuigbaren Kapazitätskontingente. Als erste nutzten Hotels in Las Vegas die Möglichkeit, über den Preis zu einer 
ertragsoptimalen Auslastung zu kommen. Traditionell sind im Hotelgewerbe die Raten über Monate (während einer definierten Haupt-/Schulter-/Nebensaison) konstant, aber die Bettenbelegung schwankt stark von Tag zu Tag. Die Hoteliers in der amerikanischen Gliicksspielmetropole dagegen setzten Preise tagesaktuell fest und erreichen so eine Auslastungsquote von ganzjährig 98\%. Ein derartiges Fine-Tuning ist mit herkömmlichen Mitteln zeitbezogener Preisdifferenzierung nicht mehr möglich.

\subsection{Yield Management}

Perfektioniert wurde die Methodik von amerikanischen Fluggesellschaften in den 80er Jahren. Ihre Systeme, die exakte Kapazitätssteuerung zur Ertragssicherung gewährleisten, waren Voraussetzung fuir flexibleres Agieren nach der Deregulierung des US-Luftverkehrs 1978 und dem Markteintritt preisaggressiver Newcomer:

Ein EDV-gesteuertes Yield Management (Yield = Ertrag, meist im Sinne von Durchschnittserlös verwendet) besteht normalerweise aus zwei Modulen für

- Prognose und

- Kontingentierung.

Das Prognosemodul liefert eine Vorschau auf die zu erwartende Buchungszahl am Tage X. Alle Erfahrungswerte der vergangenen Jahre fließen ein. So weiß man etwa, daß 20\% der Reisenden auf einer bestimmten Strecke zu einem gegebenen Kalenderdatum bis zu 70 Tage vor Abflug (-70) reservieren, weitere $30 \%$ an den Tagen -69 bis -40 und so fort. Aus dem heutigen Buchungsstand ist somit eine Abschätzung der Auslastung des betrachteten Fluges in naher Zukunft möglich. Parallel dazu lassen sich die Buchungshistorie und das reale Passagieraufkommen vergleichbarer Strecken zur Adjustierung und Absicherung der Schätzung einarbeiten. Derartige Prognosen müssen für ein wirklich funktionsfähiges Yield Management System für jeden einzelnen Flug der Gesellschaft Tag fiir Tag erstellt werden.

Das Prognosemodul liefert die Input-Daten für die Kontingentbestimmung. Hier gilt der Grundsatz, daß Buchungen zum höchsten Tarif immer akzeptiert werden, für die verbilligten Sonderpreise jedoch nur eine begrenzte Platzzahl vorgesehen ist. Diese muß heute nicht mehr starr zu Beginn der Flugplanperiode festgelegt werden, sondern kann anhand der Prognosedaten stark variieren. Sind für den billigsten Preis auf einem gegebenen Flug 6 Sitze vorgesehen und deuten schwache Buchungszahlen auf Gesamtauslastung des Fluges unter dem ursprünglich angepeilten Niveau, öffnet das System automatisch 2-4 weitere Plätze für den Minipreis. Steigen die allgemeinen Buchungszahlen in den Wochen vor Abflug dagegen stärker als im vergleichbaren Vorjahreszeitraum, so verringert sich das Kontingent der Billigtarife entsprechend - möglicherweise bis 0 .

Meist gibt es preisliche Differenzierungen in Form von drei bis vier Tarifarten mit unterschiedlichen Bedingungen (z. B. Vorausbuchung mindestens 14 Tage, Mindestaufenthalt 3 Tage etc.), die gegenüber dem Normaltarif zwischen 30 und $60 \%$ verbilligt sind. Eine komplizierte

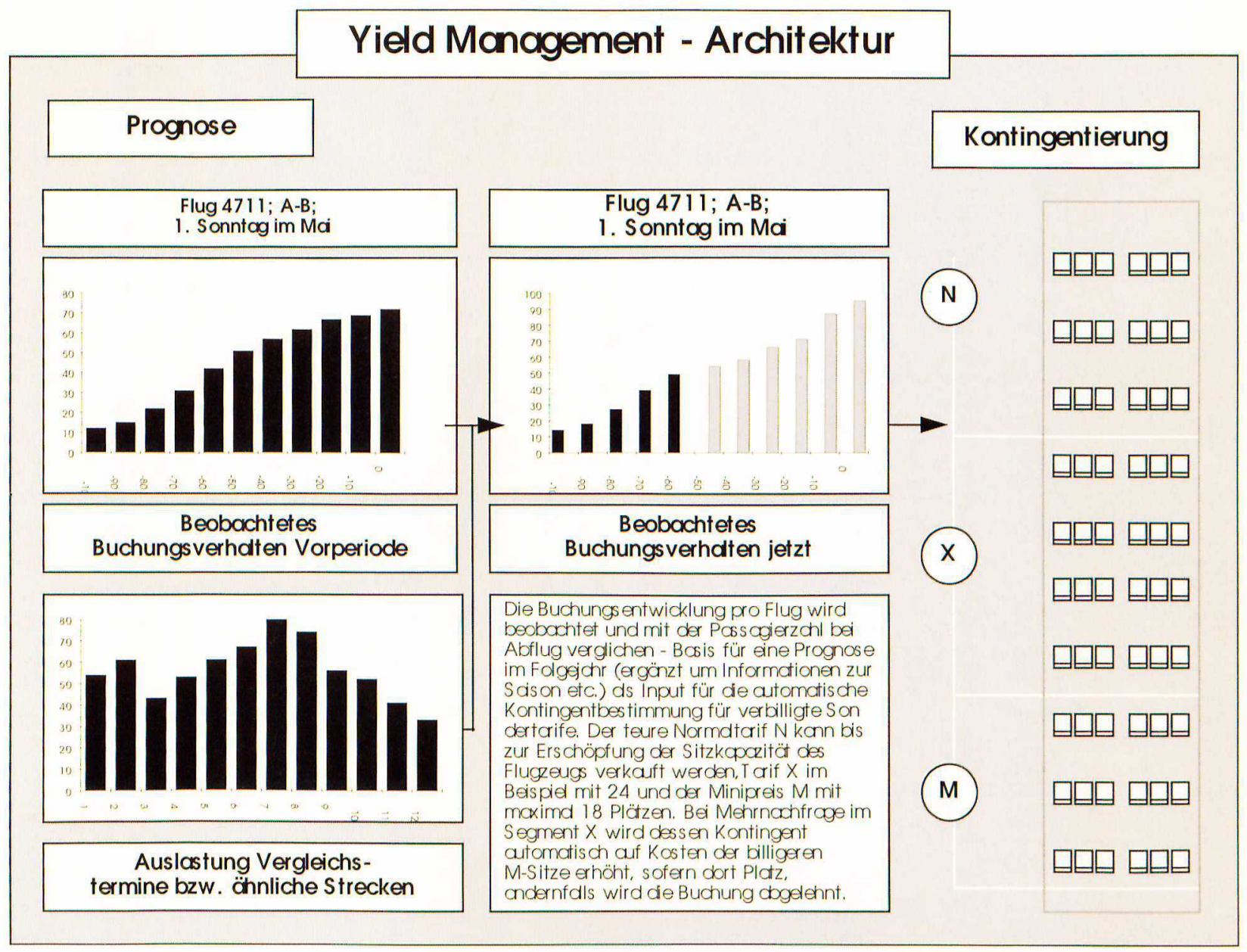


Besonderheit sind Gruppenbuchungen von Reiseveranstaltern, die sich sehr fruih um verbindliche Platzzusagen zu Vorzugskonditionen bemiihen, aber auf dem Recht zu kurzfristigem Storno bestehen, wenn sich ihre Erwartungen beim Absatz des Gesamtreisepaketes nicht erfüllen. Mittels Yield Management erhält die Fluggesellschaft die notwendigen Daten für entsprechende Preisverhandlungen und Hinweise, wie der Reiseveranstalter durch Terminmodifikation besonders vorteilhafte Flugkonditionen erwirken kann oder ob sich der Einsatz eines größeren Flugzeuges lohnt. Für Fluggesellschaften war die reine Masse an Daten lange Zeit das Haupthindernis für eine ertragsoptimierende Steuerung - GröBenordnung Lufthansa: mehr als 300000 Fliigen pro Jahr mit 28 Mio. Passagieren - die Leistungskraft der EDV hat dieses Problem gelöst.

\section{Preisbildung als unternehmerische Entscheidung}

\subsection{Organisation der Entscheidungsprozesse}

Trotz der uiberragenden Bedeutung der Absatzpreise für den Markterfolg und das Geschäftsergebnis ist die Kompetenz der Preisfestlegung keinesfalls in allen Unternehmen klar geregelt - für Dienstleister gilt dies noch mehr als für die Hersteller physischer Güiter: In der Theorie folgen die Unternehmen mehrheitlich der Regel, daß

- die preisstrategischen Linien auf der obersten Geschäftsführungsebene,

- die konkreten Preise einzelner Produkte bzw. in einzelnen Märkten vom Leiter des Verkaufsressorts,

- eventuelle Abweichungen im Einzelfall vom regionalen Vertriebsmanager

bestimmt werden. Häufig wird die Zustimmung des Controllers (bzw. des Leiters Finanz- und Rechnungswesen) zu Preisentscheidungen auf der zweiten oder dritten Ebene gefordert. Da die Grenze zwischen strategischer Linie und taktischem Scharmuitzel leicht zerfließt, sind Konflikte vorprogrammiert. Wo häufig Einzelentscheidungen unter Zeitdruck zu treffen sind, kann sich ein umständliches hausinternes Genehmigungsverfahrens bei Preiszugeständnissen zu einem spürbaren Wettbewerbsnachteil entwickeln. Dies gilt vor allem, wenn andere in Verkaufsverhandlungen wichtige Variablen (Zahlungsziele, Garantiezusagen) von anderen Instanzen zu genehmigen sind als der Preis an sich.

Der Organisation des Entscheidungsprozesses kommt daher iiberragende Bedeutung zu. Wer den Ablauf der Preis- und Konditionenfestlegung richtig fixiert hat, kann im Tagesgeschäft nervenzerrüttende Debatten über zu hohe, zu niedrige, falsch differenzierte, schlecht kommunizierte Preise im Markt vermeiden. Rezeptartige Regeln fuir alle Branchen und Situationen gibt es naturgemäß nicht, nur wenige Erkenntnisse und Erfahrungen von allgemeiner Güiltigkeit stehen zur Verfuigung.

Füir die konkreten Preisentscheidungen auf taktischem Niveau ist zwischen der Fixierung von Listenpreisen und der Aushandlung von Einzelgeschäften zu unterscheiden. Die Listenpreise legt der Verkauf normalerweise in Abstimmung mit dem Controller fest. Dabei miissen alle
Konditionen, die finanzielle Auswirkungen haben können, mit definiert werden. In traditionell funktional aufgebauten Unternehmen hat sich ein regelmäßig tagendes ressortiibergreifendes Preiskomitee bewährt. Um unauflösliche Patt-Situationen zu vermeiden, wird dieses sinnvollerweise aus drei Personen respektive Fachbereichen zusammengesetzt, Verkaufsressort und Controlling als „Partei“ und ein neutraler Sitzungsleiter mit Stimmrecht. Auf längere Sicht läßt sich die Zusammenarbeit deutlich verbessern, wenn das Controlling selbst unmittelbare Markteinsichten erwirbt, etwa indem die Mitarbeiter einige Tage lang einen Außenverkäufer bei Kundenbesuchen begleiten. Spezialisierte Preisabteilungen können sinnvoll sein, wo häufig entsprechende Entscheidungen mit Einzelfallcharakter zu fällen sind. Yield-Management Einheiten bei Fluggesellschaften und Hotelketten sind nicht nur zur Entscheidungsunterstiitzung da, sondem in erheblichem Rahmen selbst zur Preisfestlegung befugt.

\subsection{Grenzen der Dezentralisierung}

Wo Einzelverhandlungen über Preise verbreitet sind, muß die Entscheidungskompetenz innerhalb der Verkaufsorganisation klar geregelt sein. Insbesondere die regionalen Verkaufsmanager und ihre Außendienstmitarbeiter fordern häufig weite Spielräume zu Zugeständnissen bei Kundengesprächen, wie sie bei der Konkurrenz angeblich bereits iiblich seien. Durch Delegation der Preiskompetenz an die Verkaufsfront wird zweifelsohne die Reaktionsschnelligkeit des Unternehmens deutlich erhöht und die Möglichkeit der marktgerechten Differenzierung gesteigert. Außerdem steigt die Motivation des Verkaufspersonals zu guter Leistung, da die resignierende Entschuldigung marktferner Preise nicht mehr zieht. Dennoch warnen die meisten Experten vor einer weitreichenden Dezentralisierung der Preisentscheidung: der Außendienst wird zu leicht den Kundenforderungen nachgeben, Erlösverfall ist die Folge. Zudem verhält sich nicht jeder Mitarbeiter gleich, das Preisgebaren des Unternehmens wird durch Ausfransungen schnell inkonsistent und unglaubwürdig. Die wenigen in diesem Feld angestellten empirischen Untersuchungen deuten darauf hin, daß die Delegation von Preis- und Konditionenentscheidungen zur Verschlechterung der Geschäftsergebnisse führt.

Daraus läßt sich die Überlegenheit konsequent zentral festgelegter Preise ableiten, aber auch die Vermutung, daß bei Dezentralisierung von Preisgestaltungsrahmen in der Praxis häufig unüberlegt agiert wird. Wo der Außendienst beispielsweise seine Provisionen auf Umsatzbasis berechnet bekommt, kann eine Verlagerung von Preiskompetenz leicht zu Fehlverhalten führen. Nur wenn es gelingt, die Provision auf Deckungsbeitragsbasis umzustellen, kann der Spielraum ausgeweitet werden. Gleichzeitig muß die Verkäufermannschaft intensiv geschult werden, nicht nur in Verhandlungstaktik, sondern auch in die Einsicht interner betriebswirtschaftlicher Zusammenhänge vor allem auf der Kostenseite. Insbesondere die Auswirkungen der aus Verkäufersicht in ihrer Tragweite leicht unterschätzten Zugeständnisse bei Konditionen müssen deutlich gemacht werden. 
Gerade die erfolgreichsten Starverkäufer neigen aus verständlichen Grïnden dazu, ihre Tricks und Tips vor Kollegen und Vorgesetzten nicht allzu freigebig zu offenbaren. Wenn sie - speziell in schwierigen Zeiten - ihre Absatzziele nur mit übermäßigen Konzessionen einfahren können, wird deshalb auch der resultierende Ertragsverfall leicht verdeckt bleiben (siehe Marn/Rosiello 1993). Demzufolge ist bei Delegation von Verhandlungsvollmacht in Preisgesprächen unternehmensintern unbedingt auf Transparenz des Verhaltens der Mitarbeiter zu achten. Jedes Zugeständnis muß dokumentiert und einer zentralen Monitoring-Stelle zugänglich gemacht werden. Die pro Außendienstler erwirtschafteten Durchschnittserlöse sind regelmäßig zu verfolgen, um Trends und Ausreißer im Auge zu halten. Einmal zugesagtes Entgegenkommen ist kaum umkehrbar, der Schaden nur eines leichtfertig agierenden Verkäufers kann iiber Jahre die Ertragslage beschädigen. Größere Entscheidungsbefugnis auf dezentraler Ebene muß mit verstärktem Kontroll- und Sanktionenarsenal der Zentrale einhergehen - die Einsicht in diese Verknuipfung läßt uibrigens manchen Regionalmanager oder Verkaufsmitarbeiter von dem Wunsch nach mehr Freiheiten im Markt schnell abriicken.

Empowerment - die weitreichende Delegation von Handlungsvollmachten an die im Kundenkontakt stehenden Mitarbeiter der unteren Hierarchieebenen gilt als einer der Schluissel zu mehr Motivation und Kundenzufriedenheit, da auf Kundenprobleme sofort reagiert werden kann. Muß daher nicht konsequenterweise auch dem Außendienstverkäufer die Entscheidungsbefugnis zu Preisen und Konditionen iiberlassen werden? Die Grenzlinie ist dort zu ziehen, wo für das Unternehmen verbindliche vertragliche Regelungen getroffen werden. Verkaufsabschluisse sind verbindliche Vertragstransaktionen und damit - anders als Entscheidungen uiber Beschwerdereaktion, Kulanzregelungen, Eillieferung in Problemfällen - kein geeignetes Feld für ein Empowerment einzelner Belegschaftsmitglieder:

\subsection{Preissteuerung und Verkaufssteuerung}

Die technischen Möglichkeiten lassen es auf der anderen Seite heute zu, den Außendienst ergebnisorientiert zu führen. Mit Laptop-Computer ausgestattete Verkäufer können ihre Daten über Umsätze und Konditionen zum Transaktionszeitpunkt an die Zentrale überspielen. Mit dem gleichen Instrumentarium läßt sich zur Risikominimierung die Alleinverantwortlichkeit uiber den Preis in der Zentrale belassen, ohne langwierige Genehmigungsverfahren anzustoßen. Der Verkäufer fragt per EDV an, ob seine Verhandlungslinie gegeniiber dem Kunden in Ordnung geht - analog dem Bonitäts-Check bei Kreditkartenkäufen. Die angestoßene Prozedur - analog dem Yield Management der Airlines - ist weitgehend automatisierbar, beispielsweise in Abhängigkeit von der Auftragslage oder strategischen Zielsetzungen in diesem Gebiet oder mit der betroffenen Produktlinie.

Legt man die Architektur des EDV-Systems von vornherein entsprechend an, werden nicht nur die getätigten Geschäfte dokumentiert, sondern auch die abgelehnten. Diese Datenbasis wird sich als unendlich wertvoll erwei- sen, wenn über Veränderungen der Preis- und Konditionenpolitik diskutiert oder gezielte Akquisition mit Vorzugskonditionen bei einem immer knapp verlorenen Beinahe-Kunden erwogen wird.

\section{Fazit}

Viele Unternehmen verschenken durch Ungeschicklichkeiten in der Preisgestaltung für Güter und Dienstleistungen bares Geld. Sachgerechter Einsatz des preispolitischen Instrumentariums auf Basis von gesicherten Marktclaten ist nicht die Regel. Deshalb uiberrascht es kaum, daß in zahlreichen Unternehmen die Diskussion über die eigene Preisgestaltung so langwierig und wenig ergiebig ausfällt. Mit faktenorientiertem Management und einem professionell organisierten Entscheidungsprozeß ist über Optimierung der Preisgestaltung sehr schnell das Eckchen mehr Gewinnspanne herauszuarbeiten, das sonst vielen Betrieben zum Überleben im Wettbewerb fehlt.

\section{Literaturhinweise}

- Bednarczuk, Piotr; Friedrich, Joachim: Kundenorientierung ohne Marketing, eine Lösung für Dienstleistungsunternehmen; in: Absatzwirtschaft 9/1992

- Biermann, Thomas: Servicequalität und Kosteneffizienz - den Widerspruch lösen; in: Gabler's Magazin 6-7/1994

- Congram, Carole (Hrsg.): The AMA Handbook of Marketing for the Service Industries, New York 1991

- Gierl, Heribert: Eine Erklärung der Preislagenwahl bei Konsumgiitern, Berlin 1992

- Haller, Sabine: Methoden zur Beurteilung von Dienstleistungsqualität; in: Zeitschrift für betriebswirtschaftliche Forschung 1/ 1993

- Hay, Christian: Die Verarbeitung von Preisinformationen durch Konsumenten; Heidelberg 1987

- Hinterhuber, Hans: Wettbewerbsstrategie, 2. Aufl., Berlin-New York 1990

- Hölzel, Friedrich/Biermann, Thomas: Die Leistungserstellung der Lufthansa und ihre Preise; in: Lufthansa Jahrbuch 1985, Köln 1985

- Kreilkamp, Edgar: Strategisches Management und Marketing, Berlin 1987

- Marn, Michael/Rosiello, Robert: Balanceakt auf der Preistreppe; in: Harvard Manager 2/1993

- Miiller, Stefan/Kesselmann, Peter: Die Preisbereitschaft von Konsumenten bei umweltfreundlich verpackten Produkten - Ergebnisse einer Conjoint Analyse; in: Zeitschrift für betriebswirtschaftliche Forschung 3/1994

- Oi, W.: A Disneyland Dilemma. Two-Part Tariffs for a Mickey Mouse Monopoly; in: Quarterly Journal of Economics, 85 (Febr.) 1971

- Röper, Jörn: Zur Theorie der dynamischen Preispolitik, Wiesbaden 1987

- Simon, Hermann: Preismanagement; 2. Aufl., Wiesbaden 1992

- Tacke, Georg: Nichtlineare Preisbildung - Höhere Gewinne durch Differenzierung; Wiesbaden 1989

- Wiese, Harald: Das Theorie-Praxis-Paradox der Kostenrechnung aus verhandlungstheoretischer Sicht; in: Zeitschrift für betriebswirtschaftliche Forschung 6/1994

- Wilson, Robert: Nonlinear Pricing, New York-Oxford 1993

\section{Verfasser}

Prof. Dr. rer. pol. Thomas Biermann

Technische Fachhochschule Wildau

Fachbereich Betriebswirtschaft/Wirtschaftsinformatik

Tel. (0 33 75) 508-329 